\title{
Handlungsbedarfe und Reformoptionen nach der Bundestagswahl
}

Anlässlich der Bundestagswahl vom 26. September 2021 widmet sich dieses Zeitgespräch den Herausforderungen für die zukünftige Bundesregierung. Das Themenspektrum ist ähnlich vielfältig wie es die Vorstellungen der Parteien sind. Neben den Themen Digitalisierung, Dekarbonisierung sowie Aus- und Fortbildung von Fachkräften steht die Finanzpolitik im Fokus. Auch Gleichberechtigung spielt eine zentrale, im Wahlkampf häufig vernachlässigte Rolle. Welche Schritte sollte die nächste Bundesregierung tun, um den anstehenden Herausforderungen zu begegnen? Die inhaltlichen Unterschiede der Wahlprogramme von FDP und Grünen sowie mögliche Kompromisslinien werden aufgezeigt. Schließlich wird erörtert, welche Maßnahmen zur Steigerung der Produktivität ergriffen werden sollten, um den Strukturwandel - hervorgerufen durch Digitalisierung und Energiewende - zu gestalten.

Finanzpolitik in der neuen Legislaturperiode: Auf wirtschaftliche Erholung und Wachstum setzen!

Clemens Fuest, ifo Institut - Leibniz-Institut für Wirtschaftsforschung an der Universität München e. V.; Ludwig-Maximilians-Universität München.

\section{Bei der Lösung der großen Zukunftsfragen sind Marktprozesse unverzichtbar}

Christoph M. Schmidt, RWI - Leibniz-Institut für Wirtschaftsforschung; Ruhr-Universität Bochum.

Torsten Schmidt, RWI - Leibniz-Institut für Wirtschaftsforschung; Ruhr-Universität Bochum.

Wirtschaftspolitische Differenzen und mögliche Kompromisse für die nächste Bundesregierung

Jens Südekum, Düsseldorf Institute for Competition Economics (DICE).

\section{Gleichberechtigung während der Pandemie}

Bettina KohIrausch, Wirtschafts- und Sozialwissenschaftliches Institut der Hans-Böckler-Stiftung, Düsseldorf.

\section{Produktivität steigern, den Strukturwandel gestalten}

Achim Wambach, ZEW - Leibniz-Zentrum für Europäische Wirtschaftsforschung in Mannheim. 